\title{
Thymidylate synthase expression as a predictive biomarker of pemetrexed sensitivity in advanced non-small cell lung cancer
}

\author{
Cristina Chamizo ${ }^{1}$, Sandra Zazo ${ }^{1}$, Manuel Dómine², Ion Cristóbal ${ }^{2}$, Jesús García-Foncillas², Federico Rojo ${ }^{1 *}$ \\ and Juan Madoz-Gúrpide ${ }^{1 *}$
}

\begin{abstract}
Background: Although it has been suggested that a high level of thymidylate synthase (TYMS) gene expression in malignant tumors is related to reduced sensitivity to the antifolate drug pemetrexed, no direct evidence for such an association has been demonstrated in routine clinical samples from patients treated with the drug. The purpose of this study was to quantitatively assess the impact of TYMS gene expression in tumor cells as a predictor of the efficacy of pemetrexed therapy in patients with advanced non-small cell lung cancer (NSCLC) treated at our institution.

Methods: Sixty-two NSCLC patients were included in this study: 16 patients received platins-pemetrexed as first-line NSCLC, and 46 pemetrexed in monotherapy as second- or subsequent-line treatment. Total mRNA was isolated and the expression of TYMS was analyzed by RT-qPCR. TYMS levels were calibrated against expression in normal lung tissue.

Results: TYMS overexpression was detected in $61 \%$ of patients and low expression in $39 \%$. The response rate for patients with low TYMS expression was 0.29 compared with 0.03 in patients with overexpression $(P=0.025)$. A significant benefit was observed in patients with low expression both in time to progression (average TTP $=56$ vs. 23 months, $P=0.001$ ) and in overall survival (average $O S=60$ vs. 25 months, $P=0.002$ ).

Conclusions: TYMS overexpression in tumor cells correlated with a reduced response to pemetrexed-containing chemotherapy and might be used as a predictive biomarker in advanced NSCLC patients.
\end{abstract}

Keywords: Thymidylate synthase, NSCLC, Pemetrexed

\section{Background}

Pemetrexed, an analogue of folic acid (folate), is a folate antimetabolite agent that shows antitumor activity, inhibiting 3 enzymes involved in de novo purine and pyrimidine synthesis: thymidylate synthase (TYMS), dihydrofolate reductase, and glycinamide ribonucleotide formyltransferase [1]. Consequently, pemetrexed inhibits DNA and RNA biosynthesis. This agent inhibits the cellular growth of a variety of tumor types and has been approved for nonsmall cell lung cancer (NSCLC) at locally advanced and metastatic stages [2] for first- and second-line therapy.

As pemetrexed inhibits TYMS more effectively than the rest of the folate-dependent enzymes, most studies

\footnotetext{
* Correspondence: FRojo@fjd.es; JMadoz@fjd.es

${ }^{1}$ Cancer Biomarkers Research Group, Fundacion Jimenez Diaz University

Hospital Health Research Institute, UAM, Madrid, Spain

Full list of author information is available at the end of the article
}

have focused on the effects of pemetrexed on TYMS. In vitro studies have demonstrated that high baseline expression levels conferred resistance to pemetrexed [3-5]. Similarly, some clinical studies have associated elevated TYMS expression levels with poorer chemotherapeutic response to pemetrexed, including breast cancer [6], colorectal cancer [7], head and neck cancer [8], and malignant pleural mesothelioma [9]. In a large phase III study in advanced-stage NSCLC patients, survival differences were reported if favor of a cisplatin/pemetrexed regimen compared to cisplatin/gemcitabine according to histology [10]. This was explained by a previous paper showing that the baseline expression of the thymidylate synthase gene and protein were significantly higher in squamous cell carcinoma compared with adenocarcinoma $(P<0.0001)[11]$. According to some published reports, elevated expression of TYMS may be predictive of 
sensitivity to pemetrexed-based chemotherapy. However, in some cancer types, such as advanced NSCLC, this point is controversial. For this reason, we evaluated the relationship between TYMS gene expression and clinical outcome in a cohort of 62 patients with advanced NSCLC treated with a pemetrexed-based regimen at our institution. A quantitative real-time PCR (qPCR) assay was devised to determine the TYMS gene expression level. qPCR is suitable for use with mRNA from archived formalin-fixed, paraffin-embedded (FFPE) samples, as it amplifies <100-bp amplicons. Additionally, it is faster and more precise than immunohistochemistry (IHC). And it has been reported a correlation between TYMS mRNA levels and protein abundance [9]. However, both techniques must be standardized before consistent comparisons can be made when interpreting retrospective/ prospective studies.

In conclusion, TYMS overexpression correlated with response to pemetrexed and death, and a significant benefit was observed in patients with low TYMS expression, suggesting that this enzyme might be used as a predictive biomarker in advanced NSCLC patients.

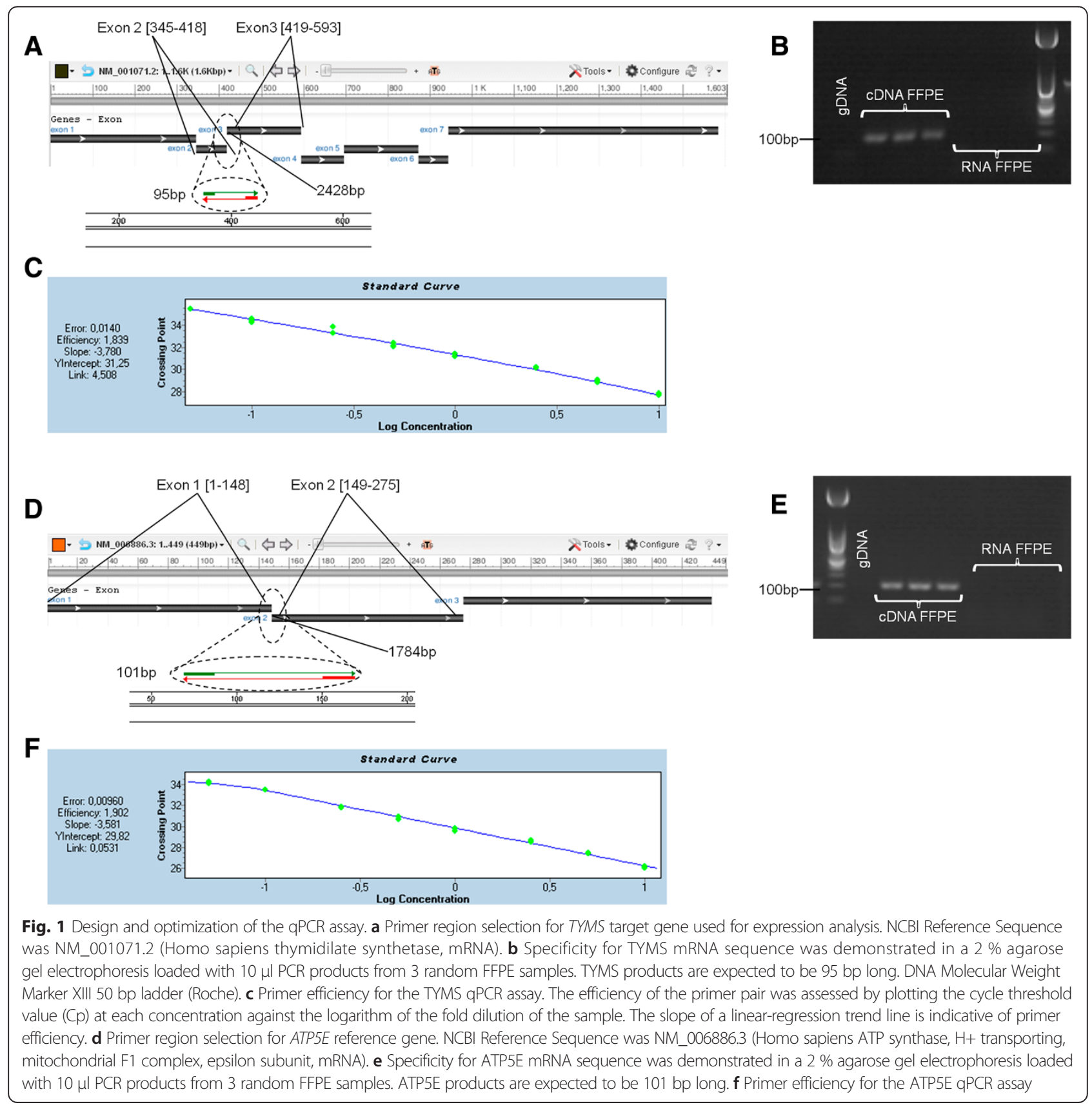




\section{Methods}

\section{Patient samples}

A single-institution retrospective analysis was carried out including samples archived in the Fundacion Jimenez Diaz Biobank (Madrid) from 62 consecutive patients who had received clinical follow-up from. The study included 62 patients with stage IV NSCLC (49 adenocarcinomas, 7 NSCLC nos and 6 squamous-cell carcinomas). Sixteen patients received platins-pemetrexed as first-line treatment for NSCLC and 46 received pemetrexed as monotherapy in second and subsequent lines. Tissue microarrays were constructed with 3 1.0-mm cores obtained from FFPE tumor biopsies before treatment. Immunostaining was performed to discriminate between histological subtypes. The study was approved by the hospital ethics committee and was conducted in accordance with institutional guidelines.

\section{Ethics statement}

The study was approved by the ethics committee of the Fundacion Jimenez Diaz hospital (CEIC-FJD) in accordance with the Spanish Royal Legislative Decree RD 223/2004.

\section{Consent statement}

Written informed consent for participation in the study was obtained from all participants.

\section{Gene expression analysis by qPCR}

The level of TYMS gene expression was determined by a quantitative RT-real time PCR assay on $5 \times 10-\mu$ m sections of the FFPE biopsies using ATP5E as a housekeeping gene. Total RNA was isolated using the RNeasy FFPE kit (Qiagen). Primers were designed according to the mRNA sequences NM_00101071 for TYMS (and NM_006886.2, and NM_001001977.1 for ATP5E. qPCRs were performed using the LightCycler480 II system (Roche Applied Science, Switzerland) for 45 cycles with the following sets of primers: TYMS, 5'-CCTCTGCTGACAACCAAACG (exon 1) and 5'-GAAGACAGCTCTTTAGCATTTG (exon 2); ATP5E, 5'-CCGGCGTCTTGGCGATTC (exon 1) and 5'-GATCTGGGAGTATCGGATG (exon 2).

Relative TYMS expression ratios were calculated using the Pfaffl method [12], using the ATP5E levels as the reference sample. TYMS expression levels were normalized to the calibrator levels (normal lung tissue) (Fig. 1). The efficiencies of every primer pair were estimated by a standard curve.

\section{Statistical analysis}

The primary end points were objective response rate, time to progression (TTP), and overall survival (OS). TTP was defined as the time from treatment to the start of progression, censored at last contact. OS was defined

Table 1 Clinical-pathological correlations for TYMS gene expression in NSCLC patients treated with pemetrexed

\begin{tabular}{|c|c|c|c|c|c|c|c|}
\hline & & \multirow{2}{*}{$\begin{array}{l}\text { Total } \\
\mathrm{n}\end{array}$} & \multicolumn{2}{|c|}{ TYMS high expression } & \multicolumn{2}{|c|}{ TYMS low expression } & \multirow[b]{2}{*}{$P$-value } \\
\hline & & & $n$ & $\%$ & $n$ & $\%$ & \\
\hline Age (mean (range)) & & $57(32-79)$ & $54(32-79)$ & & $66(60-78)$ & & 1 \\
\hline \multirow[t]{2}{*}{ Gender } & Male & 40 & 26 & 65.0 & 14 & 35.0 & 0.419 \\
\hline & Female & 22 & 12 & 54.5 & 10 & 45.5 & \\
\hline \multirow[t]{3}{*}{ Smoking habit } & Current smoker & 24 & 18 & 75.0 & 6 & 25.0 & 0.056 \\
\hline & Former smoker & 22 & 14 & 63.6 & 8 & 36.4 & \\
\hline & Never smoker & 16 & 6 & 37.5 & 10 & 62.5 & \\
\hline \multirow[t]{3}{*}{ Histology } & Adenocarcinoma & 49 & 27 & 55.1 & 22 & 44.9 & 0.071 \\
\hline & NSCLC nos & 7 & 7 & 100 & 0 & 0 & \\
\hline & Squamous cell carcinoma & 6 & 4 & 66.7 & 2 & 33.3 & \\
\hline \multirow[t]{3}{*}{ ECOG performance status } & ECOGO & 27 & 19 & 70.4 & 8 & 29.6 & 0.392 \\
\hline & ECOG1 & 32 & 17 & 53.1 & 15 & 46.9 & \\
\hline & ECOG2 & 3 & 2 & 66.7 & 1 & 33.3 & \\
\hline \multirow[t]{3}{*}{ Line of treatment } & 1st line & 16 & 9 & 56.3 & 7 & 43.8 & 0.661 \\
\hline & 2nd line & 14 & 10 & 71.4 & 4 & 28.6 & \\
\hline & 3rd, further lines & 32 & 19 & 59.4 & 13 & 40.6 & \\
\hline \multirow[t]{3}{*}{ Response } & No & 45 & 30 & 66.7 & 15 & 33.3 & 0.025 \\
\hline & Yes & 7 & 1 & 14.3 & 6 & 85.7 & \\
\hline & Not evaluatable & 10 & 7 & 70.0 & 3 & 30.0 & \\
\hline \multirow[t]{2}{*}{ Progression } & No & 24 & 4 & 16.7 & 20 & 83.3 & 0.003 \\
\hline & Yes & 38 & 34 & 89.5 & 4 & 10.5 & \\
\hline
\end{tabular}


as the time elapsed from the date of initial diagnosis to the date of death from any cause or the date of last follow-up. Receiver operating characteristic (ROC) analysis was used to determine the optimal cutoff value based on progression endpoint for TYMS. Survivals were analyzed by the Kaplan-Meier method (median followup, 75 months) and curves were compared by the logrank test. Multivariate analysis including continuous quantitative and qualitative clinical-pathologic parameters was done using the Cox proportional hazards model. All statistical tests were conducted at the two-sided 0.05 level of significance. This work was performed in accordance with the Reporting Recommendations for Tumor Marker Prognostic Studies (REMARK) guideline.
Statistical analysis was carried out using the IBM SPSS, version 21.0.

\section{Results}

\section{TYMS gene expression in advanced tumors}

In order to assess the suitability of TYMS gene-expression measurement as a predictive marker of pemetrexed sensitivity in advanced NSCLC patients, we first quantitatively evaluated the TYMS gene-expression levels in samples from the 62 pemetrexed-treated patients in our series. Patient data were normalized against healthy lung-tissue values. By performing a ROC curve analysis of TYMS expression against disease progression, we established a cutoff value of 2.55 . Interestingly, 38 patients (61 \%) were

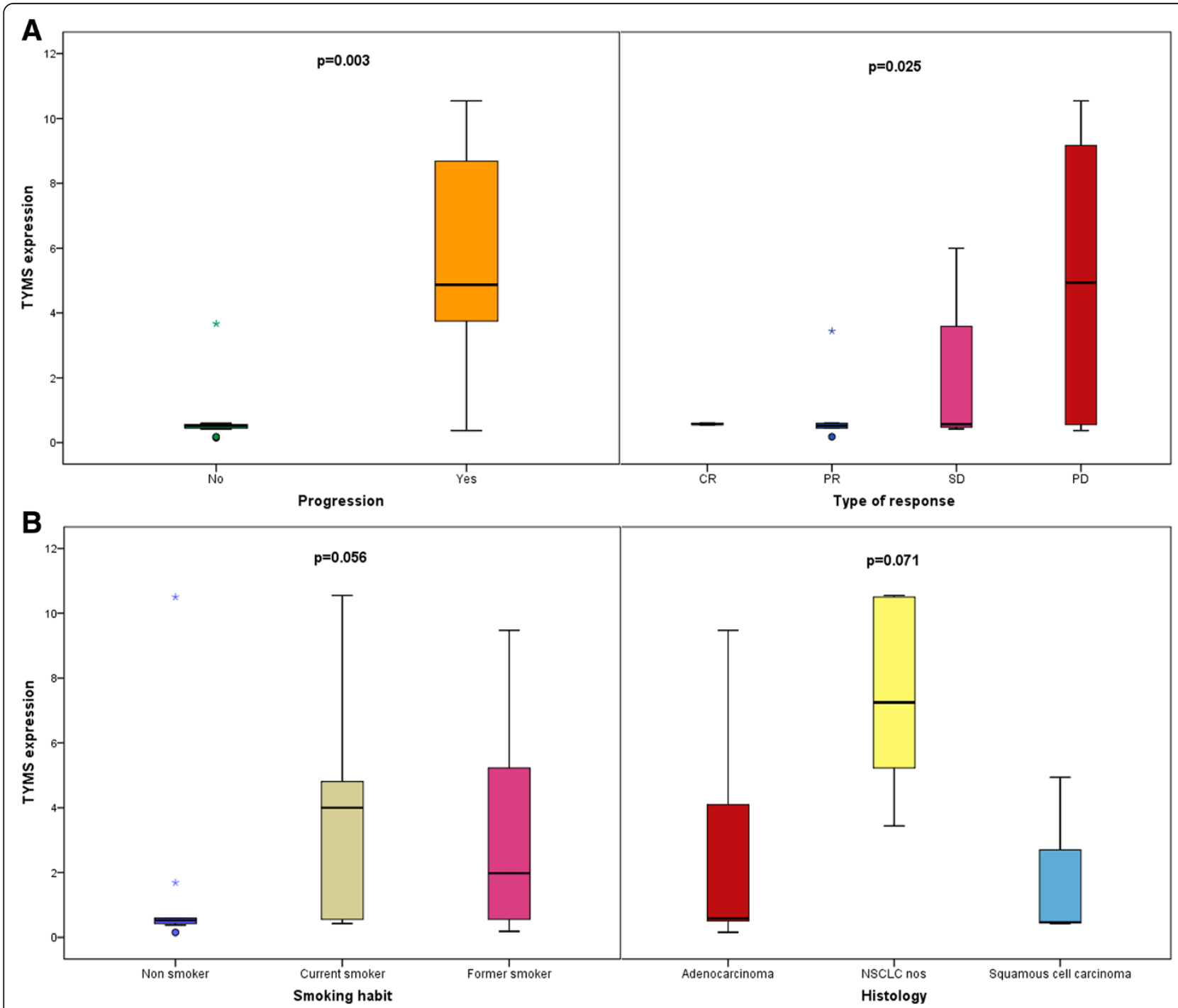

Fig. 2 a Significant clinical-pathological correlations for TYMS expression in NSCLC patients. With respect to the type of response, tumors were categorized as either responding (CR, complete response or PR, partial response) or non-responding (SD, stable disease or PD, progressive disease). b Close-to-significance correlations 
labeled as TYMS-overexpressing and 24 cases (39 \%) showed low expression.

\section{TYMS overexpression is associated with disease progression}

The clinical characteristics of the patients are shown in Table 1. TYMS gene-overexpression analysis was performed in 62 cases for which complete clinical records were available. This analysis showed significant correlation with progression $(P=0.003)$ and response to pemetrexed $(P=0.025)$ (Fig. 2a). We categorized the tumors as either responding or non-responding. The non-responding group showed significantly higher levels of TYMS expression as compared to the responding group. TYMS overexpression analysis also showed a tendency toward correlation between overexpression smoking $(P=0.056)$ and histology $(P=0.071)$ (Fig. $2 \mathrm{~b})$, but was not associated with gender, performance status, or line of treatment.

\section{Low TYMS expression levels predict delayed progression} in advanced cancers

We found that patients with a low level of TYMS gene expression (cutoff $<2.55$ ) had a significantly longer TTP than those with a high level (Fig. 3). NSCLC patients
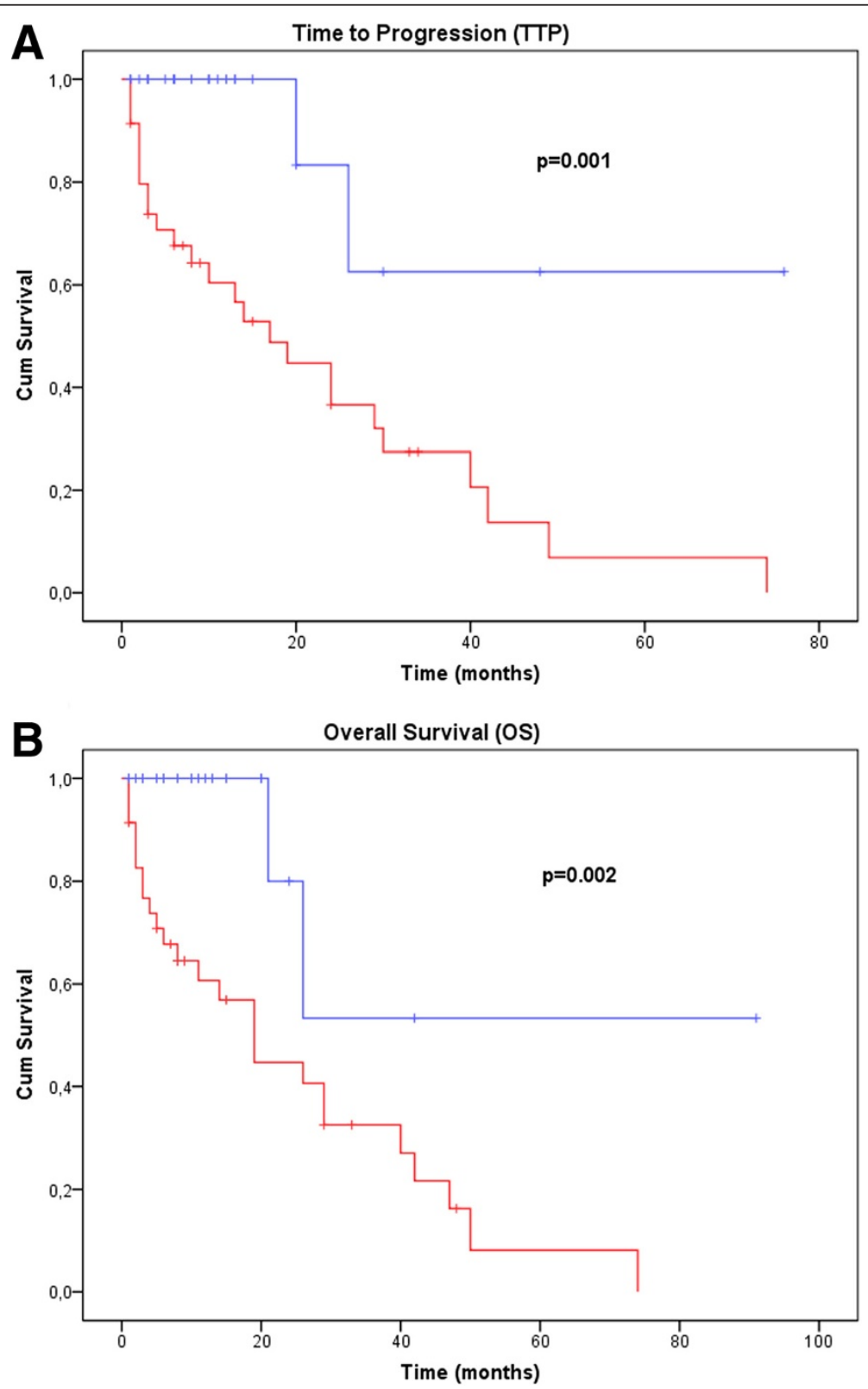

Fig. 3 TTP (a) and OS (b) in NSCLC patients. The blue line denotes patients with low TYMS gene expression; the red line indicates patients with high TYMS expression 
with low TYMS expression levels showed a significant benefit when treated with pemetrexed both in time to progression (average TTP $=56$ vs. 23 months, $P=0.001$ ) and in overall survival (average OS $=60$ vs. 25 months, $P=0.002$ ) (Fig. 3). These data thus suggest that TYMS expression level in advanced NSCLC tumors is inversely correlated with response to pemetrexed.

\section{Discussion}

In the present study, we investigated the effects of TYMS gene overexpression on the sensitivity of advanced cancer cells to pemetrexed. Reliable predictive markers of beneficial therapy can aid in determining the most appropriate therapy for patients and minimize the negative effects of certain treatment regimens in non-responsive patients.

TYMS gene expression was determined by qPCR gene expression analysis in a series of NSCLC patients. Our results confirm the overexpression of TYMS in this population and suggest that assessment of TYMS geneexpression levels by qPCR may be of predictive value when assesing sensitivity to pemetrexed-based chemotherapy in NSCLC. There is an expanding corpus of reports about TYMS expression levels in NSCLC patients, some confirming that TYMS expression is significantly increased in tumor cells but not in normal epithelial cells $[9,13]$. Recently, in accordance with the results found in our series described here, 2 meta-analyses reported that null or low expression of TYMS was associated with higher objective response in NSCLC patients treated with pemetrexed-containing chemotherapy $[14,15]$. Of note, objective response rates were significantly higher in TYMS -/low expression patients than in TYMS +/high expression patients when examined by IHC. However, this difference did not reach statistical significance in studies performed by RT-PCR [14], thus contrasting with our results. Both of the aforementioned studies suggest that TYMS may be a suitable marker of sensitivity to pemetrexed-based chemotherapy in patients with NSCLC, although one of them indicates that the prognostic value of TYMS protein expression may need further validation. In our case, TYMS overexpression correlated significantly with progression and type of response. A small number of studies have also addressed the relationship between TYMS expression and effect of pemetrexedbased chemotherapy, although reports about the prognostic significance of TYMS expression in advanced NSCLC are controversial [16]. Notably, most of them suggest that elevated levels of TYMS expression are significantly associated with reduced tumor responses and shorter survival rates $[6,9,17]$. In addition, our data illustrated that treatment with pemetrexed benefited patients with low TYMS gene expression in terms of TTP. Although other studies have suggested that TYMS expression holds potential as a predictor of responsiveness to pemetrexed treatment in advanced cancer patients $[3,6,9,18]$, prospective studies are necessary to confirm these findings in NSCLC patients.

Our study offers evidence in support of using qPCR to determine TYMS mRNA expression as an alternative to the standard evaluation of protein expression (i.e., IHC), provided at least $80 \%$ tumor cell content per sample is achieved by laser capture microdissection. Although most of the reported studies have used IHC to evaluate TYMS abundance levels, and only a minority have used qPCR, some meta-analyses have failed to find evidence of heterogeneity between detection-method subgroups (either IHC or qPCR) [14]. Moreover, another metaanalysis reported that there was a significant correlation between IHC and qPCR findings in the detection of TYMS expression and their corresponding associations with survival rates [3].

\section{Conclusions}

In conclusion, our study carried out using qPCR assay reveals that the TYMS gene was predominantly overexpressed in these routine clinical samples, and that TYMS overexpression correlated with reduced response to pemetrexed-containing chemotherapy. In light of these findings, TYMS gene expression might be used as a predictive biomarker of sensitivity to pemetrexedbased chemotherapy in advanced NSCLC patients. Further prospective studies are ongoing in our institution to validate the appropriateness of using TYMS in clinical decision making.

\section{Abbreviations}

TYMS: Thymidylate synthase; qPCR: Quantitative real-time PCR; FFPE: Formalinfixed paraffin-embedded; IHC: Immunohistochemistry; TTP: Time to progression; OS: Overall survival; ROC: Receiver operating characteristic; REMARK: Reporting Recommendations for Tumor Marker Prognostic Studies.

\section{Competing interests}

The authors declare that they have no competing interests.

\section{Authors' contributions}

Conceived and designed the experiments: CC, JGF, FR. Selected the samples: MD. Collected patient's clinical information: MD, CC, JMG. Performed the experiments: CC, SZ, IC. Analyzed the data: CC, JMG. Wrote the paper: JMG. All authors read and approved the final manuscript.

\section{Acknowledgements}

The present work was supported by grants from the Spanish Ministerio de Economia y Competitividad (MINECO) (AES Program, grant PI12/01552); the Ministerio de Sanidad (Cancer Network); and the Comunidad de Madrid (S2010/BMD-2344). The Fundacion Jimenez Diaz Biobank is funded by a grant from the MINECO (Instituto de Salud Carlos III, RETICS Red de Biobancos, with FEDER funds, RD09/0076/00101). S.Z. and C.C. are supported by grants from the same Biobanks initiative.

\section{Author details}

${ }^{1}$ Cancer Biomarkers Research Group, Fundacion Jimenez Diaz University Hospital Health Research Institute, UAM, Madrid, Spain. ${ }^{2}$ Translational Oncology Division, Oncohealth Institute, Fundacion Jimenez Diaz University Hospital, Madrid, Spain. 
Received: 5 March 2015 Accepted: 19 October 2015

Published online: 26 October 2015

\section{References}

1. Shih C, Chen VJ, Gossett LS, Gates SB, Mackellar WC, Habeck LL, et al. LY231514, a pyrrolo[2,3-d]pyrimidine-based antifolate that inhibits multiple folate-requiring enzymes. Cancer Res. 1997;57(6):1116-23.

2. Hanna N, Shepherd FA, Fossella FV, Pereira JR, De Marinis F, von Pawel J, et al. Randomized phase III trial of pemetrexed versus docetaxel in patients with non-small-cell lung cancer previously treated with chemotherapy. J Clin Oncol. 2004;22(9):1589-97.

3. Shimizu T, Nakanishi Y, Nakagawa Y, Tsujino I, Takahashi N, Nemoto N, et al. Association between expression of thymidylate synthase, dihydrofolate reductase, and glycinamide ribonucleotide formyltransferase and efficacy of pemetrexed in advanced non-small cell lung cancer. Anticancer Res. 2012;32(10):4589-96.

4. Buque A, Aresti U, Calvo B, Sh Muhialdin J, Munoz A, Carrera S, et al. Thymidylate synthase expression determines pemetrexed targets and resistance development in tumour cells. PLOS ONE. 2013;8(5):e63338.

5. Ozasa H, Oguri T, Uemura T, Miyazaki M, Maeno K, Sato S, et al. Significance of thymidylate synthase for resistance to pemetrexed in lung cancer. Cancer Sci. 2010;101(1):161-6.

6. Gomez HL, Santillana SL, Vallejos CS, Velarde R, Sanchez J, Wang X, et al. A phase $\|$ trial of pemetrexed in advanced breast cancer: clinical response and association with molecular target expression. Clin Cancer Res. 2006;12(3 Pt 1):832-8.

7. Rose MG, Farrell MP, Schmitz JC. Thymidylate synthase: a critical target for cancer chemotherapy. Clin Colorectal Cancer. 2002;1(4):220-9.

8. Johnston PG, Mick R, Recant W, Behan KA, Dolan ME, Ratain MJ, et al. Thymidylate synthase expression and response to neoadjuvant chemotherapy in patients with advanced head and neck cancer. J Natl Cancer Inst. 1997;89(4):308-13

9. Righi L, Papotti MG, Ceppi P, Bille A, Bacillo E, Molinaro L, et al. Thymidylate synthase but not excision repair cross-complementation group 1 tumor expression predicts outcome in patients with malignant pleural mesothelioma treated with pemetrexed-based chemotherapy. J Clin Oncol. 2010;28(9):1534-9.

10. Scagliotti GV, Parikh P, von Pawel J, Biesma B, Vansteenkiste J, Manegold C, et al. Phase III study comparing cisplatin plus gemcitabine with cisplatin plus pemetrexed in chemotherapy-naive patients with advanced-stage nonsmall-cell lung cancer. J Clin Oncol. 2008;26(21):3543-51.

11. Ceppi P, Volante M, Saviozzi S, Rapa I, Novello S, Cambieri A, et al. Squamous cell carcinoma of the lung compared with other histotypes shows higher messenger RNA and protein levels for thymidylate synthase. Cancer. 2006;107(7):1589-96.

12. Pfaffl MW. A new mathematical model for relative quantification in real-time RT-PCR. Nucleic Acids Res. 2001;29(9):e45.

13. Tanaka F, Wada H, Fukui Y, Fukushima M. Thymidylate synthase (TS) gene expression in primary lung cancer patients: a large-scale study in Japanese population. Ann Oncol. 2011;22(8):1791-7.

14. Wang L, Wang R, Pan Y, Sun Y, Zhang J, Chen $H$. The pemetrexed-containing treatments in the non-small cell lung cancer is -/low thymidylate synthase expression better than +/high thymidylate synthase expression: a metaanalysis. BMC cancer. 2014;14:205.

15. Liu Q, Yu Z, Xiang $Y$, Wu N, Wu L, Xu B, et al. Prognostic and predictive significance of thymidylate synthase protein expression in non-small cell lung cancer: a systematic review and meta-analysis. Cancer Biomark. 2015;15(1):65-78

16. Wang $T$, Chuan Pan $C$, Rui Yu J, Long $Y$, Hong Cai $X$, De Yin $X$, et al. Association between TYMS expression and efficacy of pemetrexed-based chemotherapy in advanced non-small cell lung cancer: a meta-analysis. PLoS ONE. 2013;8(9):e74284.

17. Takezawa K, Okamoto I, Okamoto W, Takeda M, Sakai K, Tsukioka S, et al. Thymidylate synthase as a determinant of pemetrexed sensitivity in non-small cell lung cancer. Br J Cancer. 2011;104(10):1594-601.

18. Zucali PA, Giovannetti E, Destro A, Mencoboni M, Ceresoli GL, Gianoncelli L, et al. Thymidylate synthase and excision repair cross-complementing group-1 as predictors of responsiveness in mesothelioma patients treated with pemetrexed/carboplatin. Clin Cancer Res. 2011;17(8):2581-90.

\section{Submit your next manuscript to BioMed Central and take full advantage of:}

- Convenient online submission

- Thorough peer review

- No space constraints or color figure charges

- Immediate publication on acceptance

- Inclusion in PubMed, CAS, Scopus and Google Scholar

- Research which is freely available for redistribution 